Open Access : ISSN : 1848-7718

http://www.pub.iapchem.org/ojs/index.php/admet/index

Original scientific paper

\title{
A Trend Model for Alzheimer's Mortality
}

\author{
Örjan Hallberg*
}

Hallberg Independent Research, Brattforsgatan 3, 12350 Farsta, Sweden

*Corresponding Author: E-mail: oerjan.hallberg@swipnet.se; Tel.: +46-8-605-4998

Received: July 07, 2015; Revised: August 02, 2015; Published: September 05, 2015

\begin{abstract}
In Sweden, mortality rates from Alzheimer's disease have increased since early 90's. In this study, we compared rates reported from 2006-2012 with projected trends determined previously and found a good fit. The objective of this study was to investigate if increased mortality can be modeled as a single exponential function of time lived in a new environment, where the risk of dying from Alzheimer's disease has been increased. The results demonstrated that the exponential model can be used to predict future mortalities for different scenarios, and that it can also project age-specific trends. We conclude that increasing mortality rates from Alzheimer's disease seem caused by an environmental change introduced since the 1990's. Since similar trend breaks also have been reported for different cancers, responsible authorities should seriously address this problem to pinpoint causative factors.
\end{abstract}

\section{Keywords}

Alzheimer; mortality; cell phones; model; exponential.

\section{Introduction}

In 2009, this author published a model study on Alzheimer mortality, where data up till 2006 were used to predict future mortality rates in Sweden [1]. In that study, the annual use of cell phones was found to be a good base for the model. After parameters were varied to best fit reported age-standardized mortality rates, calculated age-specific rates also perfectly matched reported data after the year 1992. In this report we wanted to see if reported mortalities since 2006 (up till 2012) have been following the projected trends predicted in the earlier report. The model work from 2009 used the multiple "Life Matrix" model to cover all birth cohorts of interest to calculate age-specific mortalities. Here, we investigated the usefulness of a much simpler exponential model, based on the time different age groups have been living older than a specific age, $P$, since a specific year $Y_{\mathrm{o}}$ when a causative environmental change started. We also wanted to project future mortality trends for different future exposure scenarios.

\section{Experimental}

Data on reported mortality due to Alzheimer's disease (AD) were retrieved from the National Board of Health and Welfare in Sweden. The "Multiple Life Matrix" model used for the first report in 2009 [1] was later also useful in trend analysis of skin melanoma, lung cancer, and brain cancer. This time we used a less complicated exponential approach, which previously worked well in modeling melanoma incidence and mortality trends [2]. 


\section{The Exponential Model}

The model assumes that the mortality can be described by an exponential function of time lived in a new environment, according to Equation (1) described below. The initial exponential increase is assumed to be similar for all age groups. Thus, it was easy to create an exponential model that applies to all ages using the following key parameters:

- $\quad A=$ age group, e.g. 70-74 years old

- $Y_{\mathrm{o}}=$ the year in which a sudden environmental change occurred

- $Y=$ calendar year

- $T=Y-Y_{0}$ : the number of years since the environmental change took place; $Y>Y_{0}$

- $P=$ age from when the environmental change has any effect, e.g. 45 years.

- $A_{\mathrm{m}}=$ Mortality in year $Y_{\mathrm{o}}$ and earlier for age group $A$

- $a=$ a constant

- $b=$ the slope used in the exponential function

The mortality $M$ is thus a function of $T$ and $A$ as given by Equation (1), formatted as an Excel IFexpression.

$$
M(T, A)=\operatorname{IF}\left(T<A-P ; A_{m}+a(\exp (b T)-1) ; A_{m}+a(\exp (b(A-P))-1)\right.
$$

We used Equation (1) to calculate the age-standardized rates $\left(A S R_{w}\right)$ of $A D$ mortality. In searching for best fit between calculated and reported rates, we varied the constant $(a)$, the exponential slope factor $(b)$, and the starting age $(P)$ to determine the best fit. To test the validity of this approach, we then compared calculated age-specific incidences with reported data without any further parameter variations. To estimate the effect of completely stopping exposure from the radiation sources involved, we used the following calculation: If the exposure is stopped in year $Y_{1}$, then the mortality at time $Y=Y_{1}+t$ for age group $A$ can be estimated according to Equation (2):

$$
M(T, A)=(A-t)_{m}+a(\exp (b(T-t))-1)
$$

where $(A-t)_{m}$ is the initial mortality before e.g. 1992 for age group A-t.

\section{Results and Discussion}

It turned out that reported age-specific mortalities from AD continued to follow the projected trends after 2006 quite well. Figure 1 shows the original graph (Figure 8 from ref [1]) as well as the reported mortality rates up until 2012.

\section{The Exponential Model}

Regular registration of AD deaths became routine in Sweden after 1987 (ICD 9). Based on the model analysis in 2009, [1], the actual mortality before 1987 was likely higher than reported at that time (Figure 1). Based on those results, we used reported rates for 1992 as $A_{m}$ in Equation (1) for each age group for the years before 1992. In 1987 cell phones operating mainly at $450 \mathrm{MHz}$ had been used for a few years. The next generation working at $900 \mathrm{MHz}$ became popular around 1992; while, the real boom occurred in late 1997 when the dual band phones, $900-1800 \mathrm{MHz}$ were introduced. To design a simple exponential model based on the time lived as an Alzheimer victim in a wireless world, we assigned 1992 as the starting year for population exposure to cell phone $\mathrm{GHz}$ radiation. Equation (1), described in Experimental section, was then used together with reported age-standardized mortality data from 1992 till 2012 to determine model parameters for the mortality trends. The reported and calculated mortality from $A D$ if the whole cell phone system closed down in 2022 (Figures 2 and 3) is an example of a scenario calculated according to Equation (2). 


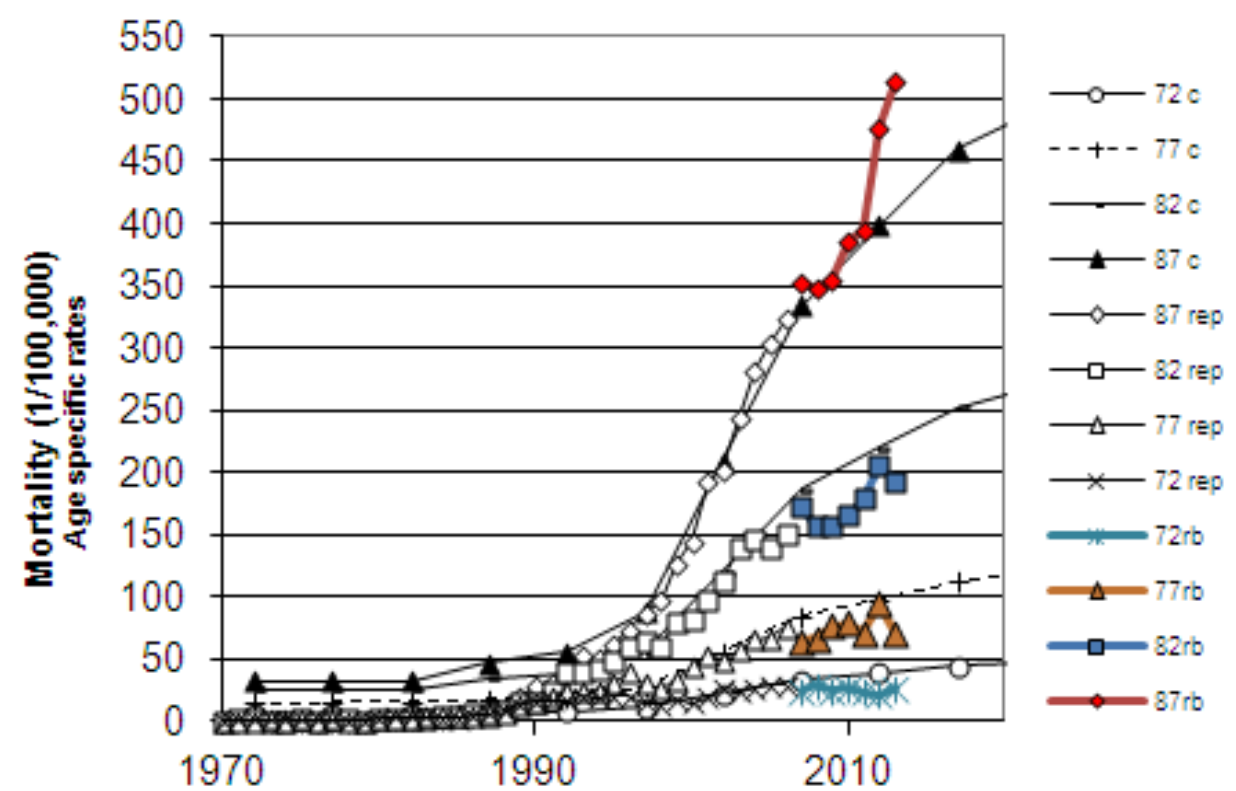

Figure 1. Reported and calculated age-specific mortality rates from AD in Sweden. Data up till 2006 are from ref [1], including the projections. Reported rates up till 2012 have here been added to the graph using filled symbols. Calculated data are marked c, reported up till 2006 by rep and reported after 2006 by rb.

The age-standardized mortalities (World standard) are calculated based on Equation (1) when all age groups are taken into account. Thus, it was easy to compare reported and calculated age-specific rates, since they had already been used to calculate age-standardized mortalities. The age-specific rates reported, as well as those calculated using the same parameters used in calculating age standardized mortalities (Figure 2, Table 1), are shown in Figure 3.

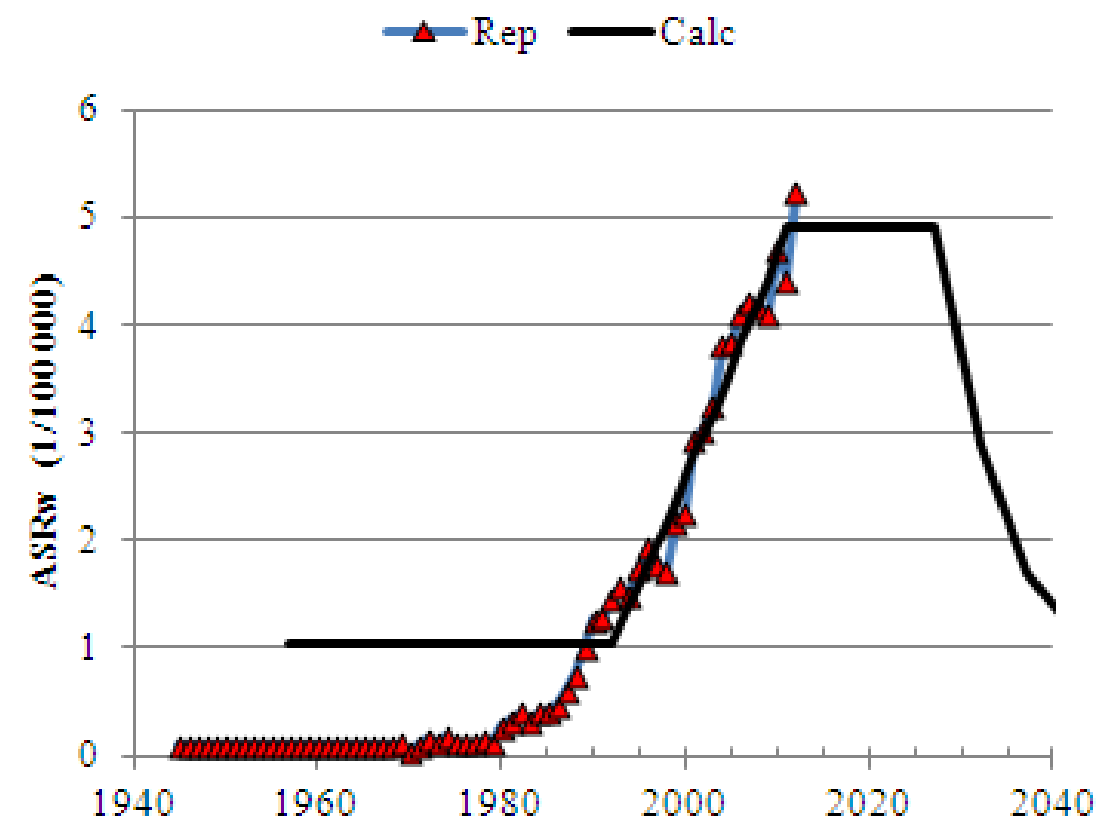

Figure 2. Reported and calculated age-standardized mortality from AD. The black line indicates the calculated total mortality if all mobile phone systems were no longer used after 2022. 
Table 1. Optimum parameters for the exponential function used in the trend models shown in Figures 2 and 3

\begin{tabular}{ccc}
\hline $\begin{array}{c}\text { Constant } \boldsymbol{a} \\
\boldsymbol{a}^{*} \exp (\boldsymbol{b} T)\end{array}$ & $\begin{array}{c}\text { Exponent } \boldsymbol{b} \\
\boldsymbol{a}^{*} \exp (\boldsymbol{b} T)\end{array}$ & $\begin{array}{c}\text { Start age } \boldsymbol{P} \\
\text { (Years) }\end{array}$ \\
\hline 25.17 & 0.1494 & 65.3 \\
\hline
\end{tabular}

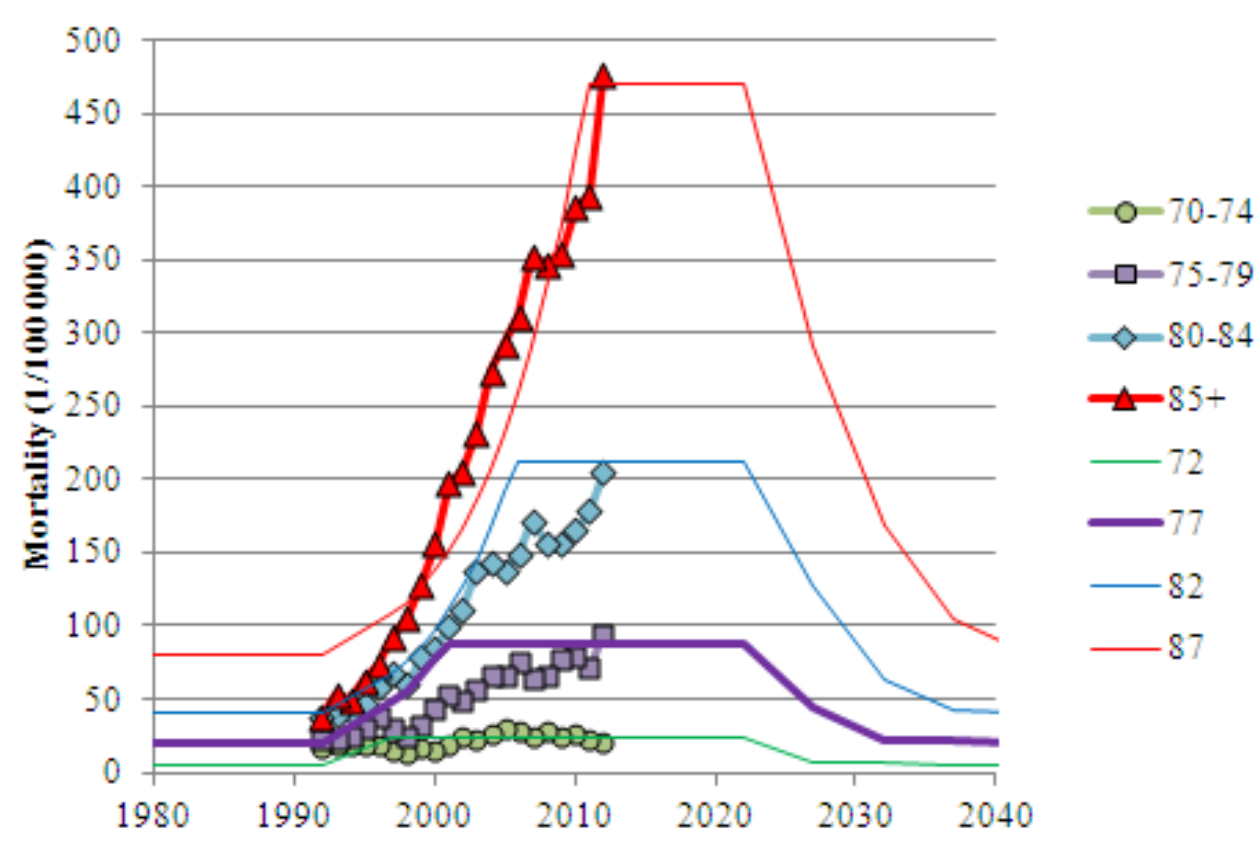

Figure 3. Reported (symbols) and calculated (lines) AD age-specific mortalities in Sweden if all cell phone systems were closed down in year 2022.

\section{Discussion}

The updated mortality data added to Figure 1 show clearly that the original model projections [1] still fit well with reported rates. Thus, we can state that the multi life matrix model seems to work well, and could be used to analyze data from other countries in a similar way. As depicted in Figure 2, increasing $A D$ mortality rates correspond with population exposure to cell phones and base station generated radiation from 1992 onwards. Figure 2 also shows how reported mortalities increased fast from 1987 to around 1990, most likely as a result of new classification standards (ICD 9) introduced in 1987. Thus, mortalities reported in 1992 were used as base level for earlier years, resulting in the flat calculated level in Figure 2 for years before 1992. The model also predicts that we will see a leveling-off of the age-standardized mortality quite soon since all age groups from 65.3 years just about now have been living in the new environment we got after 1992.

This simple exponential model also projected age-specific rates that did fit well with reported data without any further parameter adjustments, as shown in Figure 3. Parameters giving best fit between calculated and reported age-standardized mortality are shown in Table 1. Equation (1) assumes that the mortality in a specific age group A will stabilize as soon as all people in that age group have been living their lives since age $P$ in the new wireless world. Reported data show that the age group of 72 years has a stable mortality from the 1990's, while the age group 77 years flattens out a few years after year 2000 . The age groups 82 and $85+$ still have some years to wait for their stabilizations to occur. Figures 1 and 3 clearly show that the more complex model from 2009 still works [1] and gives the most accurate projections of age- 
specific mortalities. However, the much simpler exponential model seems as useful for age-standardized projections and to estimate the response to an improved environment.

Research on biological changes in the brain, which accelerate the mortality risk among AD patients, should be performed. Possible connections between continuous base station radiation, cell phone use, and biological changes should also be investigated. In 2014, V. Lauer published a theory and model about the interaction of $T$ lymphocytes with electromagnetic fields [3]. His work further contributes to the understanding of biological effects from non-ionizing radiation.

Data on AD mortality from the USA and from the 21 different Swedish counties in the time period 19992004 show that the mortality was higher in states or counties where the population density was lower $[4,5]$. Data from 2010 in the USA had an even stronger linear association $(p=0.011)$ to population density than in 1999-2004 ( $p=0.038)$, [6]. This suggests that the higher average output power from cell phone handsets in sparsely populated areas also might be an important factor to consider (Figure 4).

The fact, that mortality among dementia patients suddenly started to increase right after 1997, was noticed in a report from the Swedish National Health and Welfare Authority. No explanation was given except that doctors seemed to have become more inclined to classify deaths as due to dementia since 1987 [7].

\section{$\Delta$ Se 97-02 OUSA 99-04}

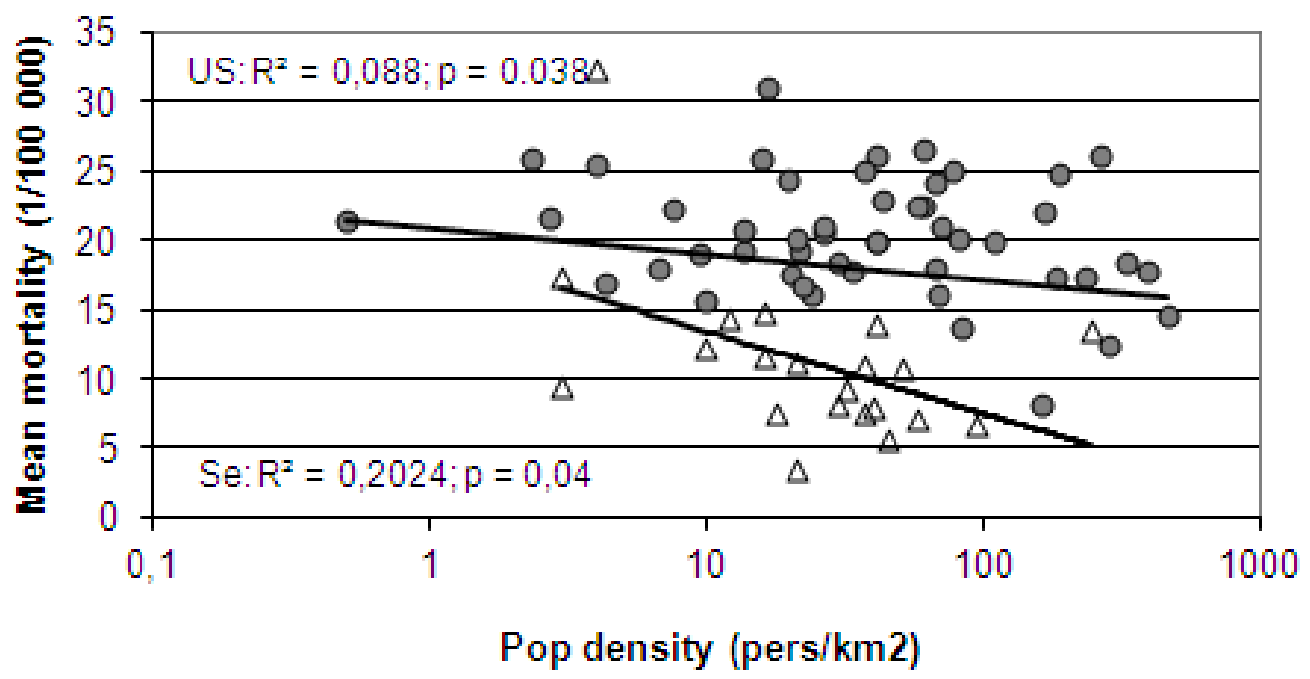

Figure 4. Mean Alzheimer mortality for men and women in Sweden (Se) and the USA vs. population density in counties (Sweden) or states (USA) during 1997-2004.

\section{Conclusions}

This short report shows that AD mortality can be modeled by different techniques with good fit to reported data. Since both models used here clearly indicate that an environmental change from around 1992 has been an important factor, responsible authorities should seriously start addressing this problem.

Acknowledgements: This study was financed by internal funds within Hallberg Independent Research. 


\section{References}

[1] Ö. Hallberg, Curr. Alzheimer Res. 6 (2009) 471-475.

[2] Ö. Hallberg, Int. J Stats. Med. Res. 4 (2015) 65-71.

[3] V. Lauer, Hyper Articles en Ligne, HAL : hal-00975963, version 1; (2014), http://hal.archivesouvertes.fr/hal-00975963.

[4] Ö. Hallberg, O. Johansson, Eur. Biol. Bioelectromag. 1 (2005) 225-246, http://sgll.nu/MedPub/Ref\%2013\%20EBB3b.pdf.

[5] K. Steenland, J. MacNeal, I. Vega, A. Levey, Alzheimer Dis. Assoc. Disord. 23 (2009) 165-170 http://www.ncbi.nlm.nih.gov/pubmed/?term=19484918.

[6] B. Tejada-Vera, Mortality from Alzheimer's disease in the United States. Data for 2000 and 2010. NCHS data brief, no 116. Hyattsville, MD: National Center for Health Statistics, (2013), http://www.ncbi.nlm.nih.gov/pubmed/23742787.

[7] OFFICIAL STATISTICS OF SWEDEN, Statistics - Health and Medical Care, Causes of Death 2012. ISBN 978-91-7555-090-9, Article nr 2013-8-6. (Figure 22),

http://www.socialstyrelsen.se/Lists/Artikelkatalog/Attachments/19175/2013-8-6.pdf.

(C)2015 by the authors; licensee IAPC, Zagreb, Croatia. This article is an open-access article distributed under the terms and conditions of the Creative Commons Attribution license (http://creativecommons.org/licenses/by/3.0/) (cc) E EY 